\title{
Exercise related transient abdominal pain
}

\section{P Morton}

\section{The causes of exercise related transient abdominal pain remain to be elucidated}

S titch, side ache, and side cramp are terms that have been used to describe an undesirable accompaniment to exercise that is well known but poorly understood. Whereas references to the pain can be found in the works of Shakespeare and Pliny the Elder, the complaint has received little attention in the medical literature. In fact, until a recent spate of investigations, no empirical studies of the pain had been published since 1951 .

\section{"The pain is mostly described as sharp or stabbing when severe, and cramping, aching, or pulling when less intense."}

Indeed, there is a wealth of anecdotal information relating to aspects of the condition, such as its symptoms, provocation, treatment, and cause. In a recent publication, in which the ailment was labelled exercise related transient abdominal pain (ETAP), an epidemiological approach was adopted to explore the various and diverse explanations of the pain. ${ }^{2}$ ETAP was shown to be remarkably similar in its manifestation when described by symptomatic people involved in various sporting activities. Hence, ETAP appears to be a single condition, rather than a variety of pains, and has been defined as a well localised pain that may occur in any region of the abdomen, but is most common in the lateral aspects of the mid abdomen. The pain is mostly described as sharp or stabbing when severe, and cramping, aching, or pulling when less intense. The condition is most prevalent in activities involving repetitive torso movement, especially when the torso is extended, and it is exacerbated by the postprandial state. ETAP is also most common in the young, and its experience may be associated with shoulder tip pain.

Although transient in nature, the experience of ETAP is widespread and can be debilitating to exercise performance. Retrospective reports indicate that over $60 \%$ of runners have experienced ETAP within the past year. ${ }^{2}$ The condition is also commonly observed in team sports, swimming, and horse riding. ${ }^{2}$ In a study of participants in a $14 \mathrm{~km}$ community run/walk, $31 \%$ reported symptoms of ETAP, and $42 \%$ of these claimed that their performance suffered as a result. ${ }^{4}$ Gastrointestinal studies in which the prevalence of ETAP is noted consistently observe ETAP to be the most prolific gastrointestinal complaint during exercise. $^{56}$

The two causes of ETAP that have traditionally featured in the literature are diaphragmatic ischaemia ${ }^{7}$ and stress on the subdiaphragmatic ligaments supporting the abdominal viscera. ${ }^{1}$ However, recent findings have proposed challenges to both theories. Evidence against a diaphragmatic origin of ETAP include the high prevalence of the complaint among horse riders, an activity characteristically not of high respiratory demand, as well as the distribution of the pain as low in the abdomen as the iliac and hypogastric regions. Further, it has been shown that spirometry measures remain unchanged during an episode of ETAP. The observed variability in the site of the pain is similarly inconsistent with the visceral ligament theory as is the observation of the pain when swimming, which lacks the torso "jolting" dynamics pivotal to the theory. In addition, the visceral innervation of the ligaments seems at variance with the well localised nature of ETAP, as the characteristics of ETAP suggest a somatic origin. These observations have led to the development of an alternative explanation for the ailment.

We have argued that characteristics of ETAP are consistent with irritation of the parietal peritoneum. ${ }^{2}$ The parietal peritoneum is sensitive to any torso movement when irritated, and as it extends throughout the abdomen it can give rise to well localised pain in various sites. ${ }^{9}$ Further, the subdiaphragmatic portion of the parietal peritoneum is supplied by branches of the phrenic nerve, which can account for the observation of shoulder tip pain in association with ETAP. The mechanism of irritation is speculative, but we have proposed that exacerbated friction may be responsible. ${ }^{2}$

Friction could be the result of increased pressure on the tissue, as in the case of a viscus being distended such as after a meal, or as a consequence of changes in the volume or properties of the serous fluid contained within the peritoneal cavity. Interestingly, clearance of the serous fluid from the peritoneal cavity is increased by diaphragmatic excursion. $^{10}$

Obviously the ultimate objective is to develop strategies for the prevention and/or treatment of ETAP. Some recent observations are that ingesting energy dense, hypertonic fluids are more provocative of ETAP than isotonic and hypotonic beverages. ${ }^{11}{ }^{12}$ Hence, these fluids should be avoided as a prevention strategy. Also, the spine may play a role in the provocation of ETAP, as we have observed that people with increased kyphosis are more susceptible to the pain, ${ }^{13}$ and ETAP can be reproduced in some people through palpation proximal to specific facet joints. ${ }^{14}$

These findings may indicate that efforts to optimise spinal integrity may be important in the management of ETAP. Indeed, many questions relating to ETAP remain unanswered and numerous research opportunities exist. Although ETAP is an old complaint, its study is relatively new. I hope that increased research attention in the future will provide clearer insights into the cause of the pain as well as innovative strategies for its management.

\section{Br J Sports Med 2003;37:287-288}

\section{Authors' affiliations}

D P Morton, Avondale College, Cooranbong, NSW, Australia

Correspondence to: Dr Morton, Avondale College, PO Box 19, Cooranbong, NSW 2265, Australia: darren.morton@avondale.edu.au

\section{REFERENCES}

1 Sinclair JD. Stitch: the side pain of athletes. N Z Med J 1951:50:607-12.

2 Morton DP, Callister R. Characteristics and etiology of exercise-related transient abdominal pain. Med Sci Sports Exerc 2000;32:432-8.

3 Morton DP, Callister R. Factors influencing exercise-related transient abdominal pain. Med Sci Sports Exerc 2002;34:745-9.

4 Morton DP, Richards D, Callister R. Epidemiology of 'stitch' at a community run/walk event [abstract]. Medi Sci Sports Exerci. 1999;35(suppl 1272):S262.

5 Koistinen PO, Janhonen P, Lehotola J, et al. Gastrointestinal symptoms during endurance running. Scand J Med Sci Sports $1991 ; 1: 232-4$

6 Rehrer NJ, Brouns F, Beckers EJ, et al. Physiological changes and gastrointestinal symptoms as a result of untra-endurance running. Eur J Appl Physiol 1992;64:1-8.

7 Capps RB. Causes of the so-called side ache in normal persons. Arch Intern Med 1941;68:94-101.

8 Morton DP, Callister R. Electromyography and spirometry measurements during 'stitch' [abstract]. Fifth International Olympic Committee World Congress on Sport Sciences: book of abstracts Sydney, October 1999. ACT: Sports Medicine Australia, 1999:226.

9 Capps JA, Coleman GH. Experimental observations on the localisation of pain sense 
in the parietal and diaphragmatic peritoneum. Arch Intern Med 1922;30:778-89.

10 Bettendorf $\mathbf{U}$. Lymph flow mechanism of the subperitoneal diaphragmatic lymphatics. Lymphology 1978;11:111-16

11 Plunkett BT, Hopkins WG. Investigation of the side pain 'stitch' induced by running after fluid ingestion. Med Sci Sport Exerc 1999;31:1169-75.
12 Morton DP, Aragon-Vargas LF, Callister R. Effect of ingested fluid composition on the experience of exercise-related transient abdominal pain. Proceedings of the Australian Conference of Science and Medicine in Sport. Perth, October. ACT: Sports Medicine Australia, 2001:63.

13 Morton DP, Aune T, Callister R. Influence of body type and posture on the experience of 'stitch' [abstract]. Fifth International Olympic
Committee World Congress on Sport Sciences: book of abstracts, Sydney, October 1999. ACT: Sports Medicine Australia 1999:226.

14 Morton DP, Aune T. Role of the thoracic spine in the experience of exercise-related transient abdominal pain. Proceedings of the Australian Conference of Science and Medicine in Sport. Perth, October. ACT: Sports Medicine Australia, 2001:80

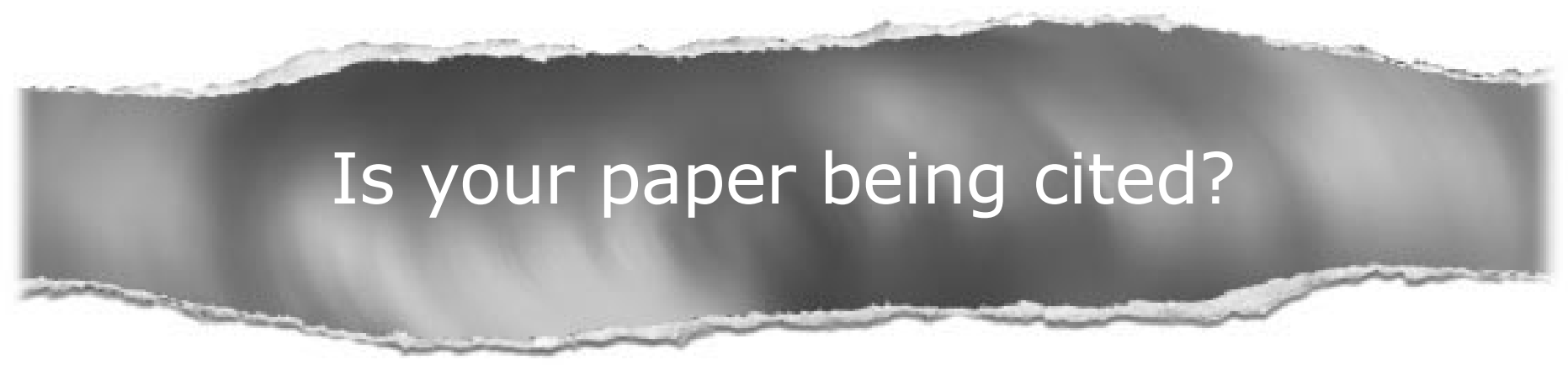

\section{CiteTrack service}

CiteTrack will alert you by email whenever new content in the British Journal of Sports Medicine or a participating journal is published

that matches criteria you want to track

Topics: Tell CiteTrack which words or subjects to watch for in new content

Authors: Be alerted whenever key authors you are following publish a new paper

Articles: Know whenever a paper of interest to you is referenced by another paper

\section{www.bjsportmed.com}

